\title{
Numerical Decomposition of Affine Algebraic Varieties
}

\section{Shawki AL Rashed and Gerhard Pfister}

\begin{abstract}
An irreducible algebraic decomposition $\cup_{i=0}^{d} X_{i}=\cup_{i=0}^{d}\left(\cup_{j=1}^{d_{i}} X_{i j}\right)$ of an affine algebraic variety $X$ can be represented as a union of finite disjoint sets $\cup_{i=0}^{d} W_{i}=\cup_{i=0}^{d}\left(\cup_{j=1}^{d_{i}} W_{i j}\right)$ called numerical irreducible decomposition (cf. [14],[15],[18],[19],[20],[22],[23],[24]). The $W_{i}$ correspond to the pure i-dimensional components $X_{i}$, and the $W_{i j}$ present the idimensional irreducible components $X_{i j}$. The numerical irreducible decomposition is implemented in BERTINI (cf. [3]). The algorithms use homotopy continuation methods. We modify this concept using partially Gröbner bases, triangular sets, local dimension, and the so-called zero sum relation. We present in this paper the corresponding algorithms and their implementations in Singular (cf. [8]). We give some examples and timings, which show that the modified algorithms are more efficient if the number of variables is not too large. For a large number of variables BERTINI is more efficient*.
\end{abstract}

\section{Introduction}

Given a system of $\mathrm{n}$ polynomials in $\mathbb{C}^{N}$,

$$
f\left(x_{1}, \ldots, x_{N}\right):=\left(\begin{array}{c}
f_{1}\left(x_{1}, \ldots, x_{N}\right) \\
\cdot \\
\cdot \\
f_{n}\left(x_{1}, \ldots, x_{N}\right)
\end{array}\right) .
$$

Key Words: Witness point set, Homotopy function, Gröbner basis, Local dimension, Monodromy action, Zero Sum Relation.

2010 Mathematics Subject Classification: Primary 14Q15.

Received: September, 2012.

Revised: September, 2012.

Accepted: February, 2013. 
Let $X=V(f)$ be the algebraic variety defined by the system above. $X$ has a unique algebraic decomposition into $d$ pure i-dimensional components $X_{i}$, $X=\cup_{i=0}^{d} X_{i}$. Where $X_{i}=\cup_{j_{i}}^{d_{i}} X_{i j}$ is empty or the union of $d_{i}$ i-dimensional irreducible components.

The numerical irreducible decomposition (cf. [15],[18],[19],[20],[23]) is given as the union $W=\cup_{i=0}^{d} W_{i}=\cup_{i=0}^{d}\left(\cup_{j=1}^{d_{i}} W_{i j}\right)$. The $W_{i}$ are called i-witness point sets and are given as an intersection of the pure i-dimensional component $X_{i}$ of $X$ with a generic linear space $L$ in $\mathbb{C}^{N}$ of dimension $N-i$. The finite sets $W_{i j}$ are called the irreducible witness point sets representing the irreducible components $X_{i j}$ of dimension $i$. The irreducible witness point sets have the following properties:

1. $W_{i j} \subset X_{i j}$,

2. $\sharp\left(W_{i j}\right)=\operatorname{deg}\left(X_{i j}\right)$,

3. $W_{i j} \cap W_{i l}=\emptyset$ for $j \neq l$.

The computation of the numerical irreducible decomposition uses numerical homotopy continuation methods (cf. [25],[26]). This requires that the number $n$ of polynomials of a given polynomial system is equal to the number $N$ of variables.

The numerical irreducible decomposition proceeds in three steps:

The first step reduces the polynomial system to a system of $N$ polynomials in $N$ variables and computes a finite set $\widehat{W}_{i}$ called witness point super set for each non-empty pure i-dimensional component $X_{i} . \widehat{W}_{i}$ consists of points of $X_{i}$ and $J_{i}$ a set of points on components of larger dimension, the so-called junk point set (cf. [15],[18],[23]).

The second step removes the points of $J_{i}$ from $\widehat{W}_{i}$ to obtain a subset $W_{i}$ of the pure i-dimensional component $X_{i}$ (cf. [23]).

The third step breakups $W_{i}$ into irreducible witness point sets representing the i-dimensional irreducible components of $X$ (cf. [14],[22]).

In $[15],[18],[23]$ the cascade algorithm is used to compute the witness point super sets $\widehat{W}_{i}$. In the second section we modify this algorithm replacing the use of the homotopy function by Gröbner basis computations at certain levels. In [23] the parameter continuation for polynomial systems is used to remove junk points from $\widehat{W}_{i}$ to obtain the i-witness point set $W_{i}$. In the third section we give a modified algorithm using local dimension, Gröbner bases in the zero-dimensional case, and the homotopy function to compute the i-witness 
point set $W_{i}$. The breakup of the witness point set $W_{i}$ into irreducible witness point sets is achieved using two algorithms (cf. [14],[22]). The first algorithm computes the points on the same irreducible component in the witness point set using path tracking techniques. The second algorithm computes a linear trace for each component which certifies the decomposition. In the fourth section we explain how to use the zero sum relation (cf. [7]) and the monodromy action on the algebraic variety to breakup $W_{i}$ into irreducible witness point sets. In the last section we give examples and timings to compare the implementations of BERTINI and Singular .

\section{Witness Point Super Set}

Definition 2.1. Let $Z=V(f)$ be an affine algebraic variety in $\mathbb{C}^{N}$ of dimension $d$, and $X$ be a pure $i$-dimensional component of $Z$. Let $L_{i}$ be a generic linear space in $\mathbb{C}^{N}$ of dimension $N-i$. A finite set $\widehat{W}_{i} \subset \mathbb{C}^{N}$ is called $i$-witness point super set for $X$ if

$$
X \cap L_{i} \subset \widehat{W}_{i} \subset Z \cap L_{i}
$$

The union $\widehat{W}$ of all $i$-witness point super sets is called a witness point super set for $Z$.

The following algorithm computes a witness point super set.

Remark 2.1. With the notations of the algorithm the following facts prove its correctness and explain our modification:

1. The positive dimensional irreducible components of $V\left(F_{1}, \ldots, F_{n}\right)$ are the same as the positive dimensional irreducible components of $V\left(f_{1}, \ldots, f_{N}\right)$. Isolated points of $V\left(F_{1}, \ldots, F_{n}\right)$ are isolated points of $V\left(f_{1}, \ldots, f_{N}\right)$.

2. $V\left(f_{1}, \ldots, f_{N}\right)$ has no components of dimension smaller then $r:=N-$ $\operatorname{rank}(f)$ (cf. [23]). Therefore the modified algorithm starts in dimension $r$.

3. Since $V\left(f_{1}, \ldots, f_{N}\right)$ is of dimension $d$, the witness point super sets in dimension greater than $d$ are empty. Therefore the modified algorithm can stop at dimension $d$.

4. For $i=0,1, \ldots, d$, the sets $\widehat{W}_{i}$ are witness point super sets for the pure $i$-dimensional components of $V\left(F_{1}, \ldots, F_{n}\right)$ (cf. [15],[23],[24]). 


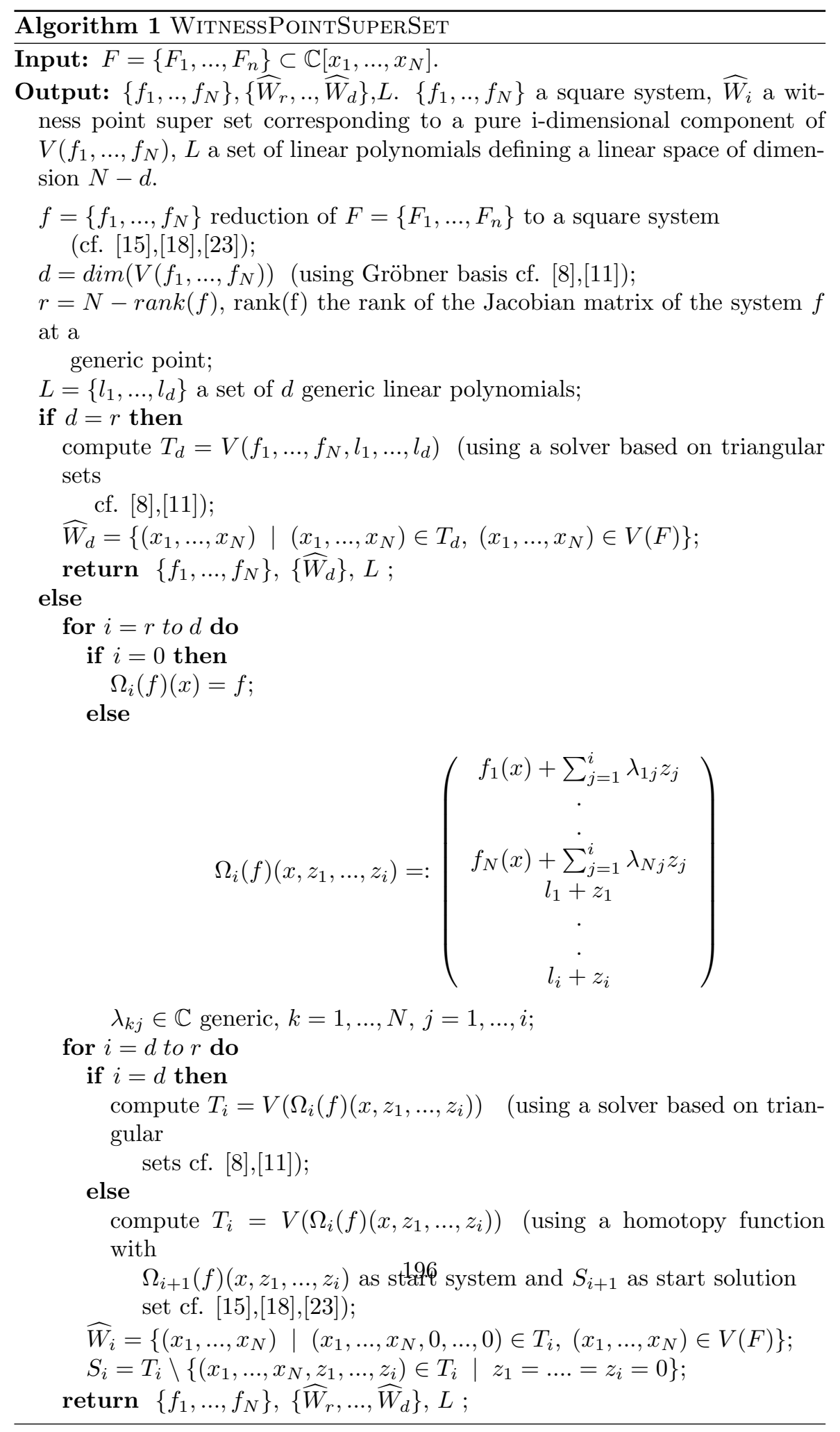


5. In [15],[18],[23] the cascade algorithm is used to compute $\widehat{W}_{i}$. It starts with $i=N-1$ to compute the witness point super sets $\widehat{W}_{i}$. It needs to define a start system $G(x)=0$ for the homotopy continuation method (cf. [25],[26]), and to know its solutions. We use a Gröbner basis of the ideal defining $Z$ to compute the dimension $d$ of $Z$, then use the cascade algorithm which starts with $i=d-1$. We will show that we do not need to define a start system.

We will illustrate our modifications of the algorithm by an example.

Example 2.1. Let $X$ be the algebraic variety defined by the polynomial system

$$
f(x, y, z)=\left(\begin{array}{c}
\left(x^{3}+z\right)\left(x^{2}-y\right) \\
\left(x^{3}+y\right)\left(x^{2}-z\right) \\
\left(x^{3}+z\right)\left(x^{3}+y\right)\left(z^{2}-y\right)
\end{array}\right) .
$$

The dimension of $X \subset \mathbb{C}^{3}$ is 1 .

The algorithm in ([15],[23]) starts at level 2, while the modified algorithm starts at level 1 to compute the witness point super set $\widehat{W}$ for the algebraic variety $X$ as follows.

- $L=\left\{l_{1}=x+y+z-1\right\}$ the set of 1 generic linear polynomials;

$$
\Omega_{0}(f)(x, y, z):=f(x, y, z)=\left(\begin{array}{c}
\left(x^{3}+z\right)\left(x^{2}-y\right) \\
\left(x^{3}+y\right)\left(x^{2}-z\right) \\
\left(x^{3}+z\right)\left(x^{3}+y\right)\left(z^{2}-y\right)
\end{array}\right)
$$

- $\lambda_{11}:=1, \lambda_{12}:=5, \lambda_{13}:=18$,

$$
\Omega_{1}(f)\left(x, y, z, z_{1}\right):=\left(\begin{array}{c}
\left(x^{3}+z\right)\left(x^{2}-y\right)+z_{1} \\
\left(x^{3}+y\right)\left(x^{2}-z\right)+5 z_{1} \\
\left(x^{3}+z\right)\left(x^{3}+y\right)\left(z^{2}-y\right)+18 z_{1} \\
x+y+z-1+z_{1}
\end{array}\right)
$$

- compute $T_{1}=\left\{t_{1}, \ldots, t_{29}\right\} \subset \mathbb{C}^{4}$ the set of solutions of $\Omega_{1}(f)\left(x, y, z, z_{1}\right)$ using the library "solve.lib" in Singular ;

- $\widehat{W}_{1}=\left\{w_{1}, \ldots, w_{7} \mid \quad \exists t_{i} \in T_{1}: t_{i}=\left(w_{i}, 0\right), i=1, \ldots, 7\right\} \subset \mathbb{C}^{3}$ the 1-witness point super set corresponding to the pure 1-dimensional component of $X$;

- $S_{1}=T_{1} \backslash\left\{\left(x, y, z, z_{1}\right) \in T_{1} \mid z_{1}=0\right\}$; 
- using the homotopy function technique, (implemented in BERTINI),

$$
T_{0}=V\left(t . \Omega_{1}(f)\left(x, y, z, z_{1}\right)+(1-t) \cdot\left(\begin{array}{c}
\Omega_{0}(f)(x, y, z) \\
z_{1}
\end{array}\right)\right),
$$

with the start system $\Omega_{1}(f)\left(x, y, z, z_{1}\right)$ and the start solution set $S_{1}$ as $t$ goes from 1 to 0 .

- $\widehat{W}_{0}=T_{0} \subset \mathbb{C}^{3}$ the 0-witness point super set corresponding to the pure 0-dimensional component of $X$;

- $\widehat{W}=\left\{\widehat{W}_{0}, \widehat{W}_{1}\right\}$ the witness point super set for $X$.

We note that we did not need to define a start system (with given solutions) to compute the witness point super set.

\section{Computation Witness Point Set}

The witness point super set $\widehat{W}_{i}$ is a union of an i-witness point set $W_{i}$ and a junk point set $J_{i}$ (cf. [15],[18],[23]),

$$
\widehat{W}_{i}=W_{i} \cup J_{i}, \quad W_{i} \subset X_{i} \text { and } J_{i} \subset \cup_{j>i} X_{j} \text { for } i=0,1, \ldots, d .
$$

We use Gröbner bases, triangular sets, local dimension and homotopy continuation method in the algorithm below to remove the points of $J_{i}$ from $\widehat{W}_{i}$ as follows.

Proof (The correctness of the Algorithm 2).

The witness point super set $\widehat{W}_{i}$ is the union of points on the i-dimensional component and points on components of dimension greater then $i . \widehat{W}_{d}$ has no junk points, i.e. $W_{d}:=\widehat{W}_{d}$. From the definition of the witness point set it follows that $s_{d}:=\sharp W_{d}$ is the degree of the d-dimensional component of $V\left(f_{1}, \ldots, f_{n}\right)$. The witness point super sets are computed numerically, that means $w \in \widehat{W}_{i}$ is an approximate value of a point $v$ on $X$. Let $Z \subset \mathbb{C}^{N} \times \mathbb{C}^{N}$ be the algebraic variety defined by the polynomial system $\left\{f_{1}-t_{1}, \ldots, f_{N}-t_{N}\right\}, y:=\left(y_{1}, \ldots, y_{N}\right) \in \mathbb{C}^{N}$ with $\|y\| \leq 10^{-16}$. Define the $\operatorname{map} \varphi: Z \subset \mathbb{C}^{N} \times \mathbb{C}^{N} \rightarrow \mathbb{C}^{N}$ by $\varphi(x, y)=y$. Then we have

$$
Z_{\varphi(v, 0)}=V\left(f_{1}, \ldots, f_{N}\right) \subset \mathbb{C}^{N} \text { and }
$$

\footnotetext{
${ }^{\dagger} w$ is the numerical approximate solution of the system $f=\left\{f_{1}, \ldots, f_{n}\right\}$, i.e. we consider $f(w)=0$ numerically.
} 


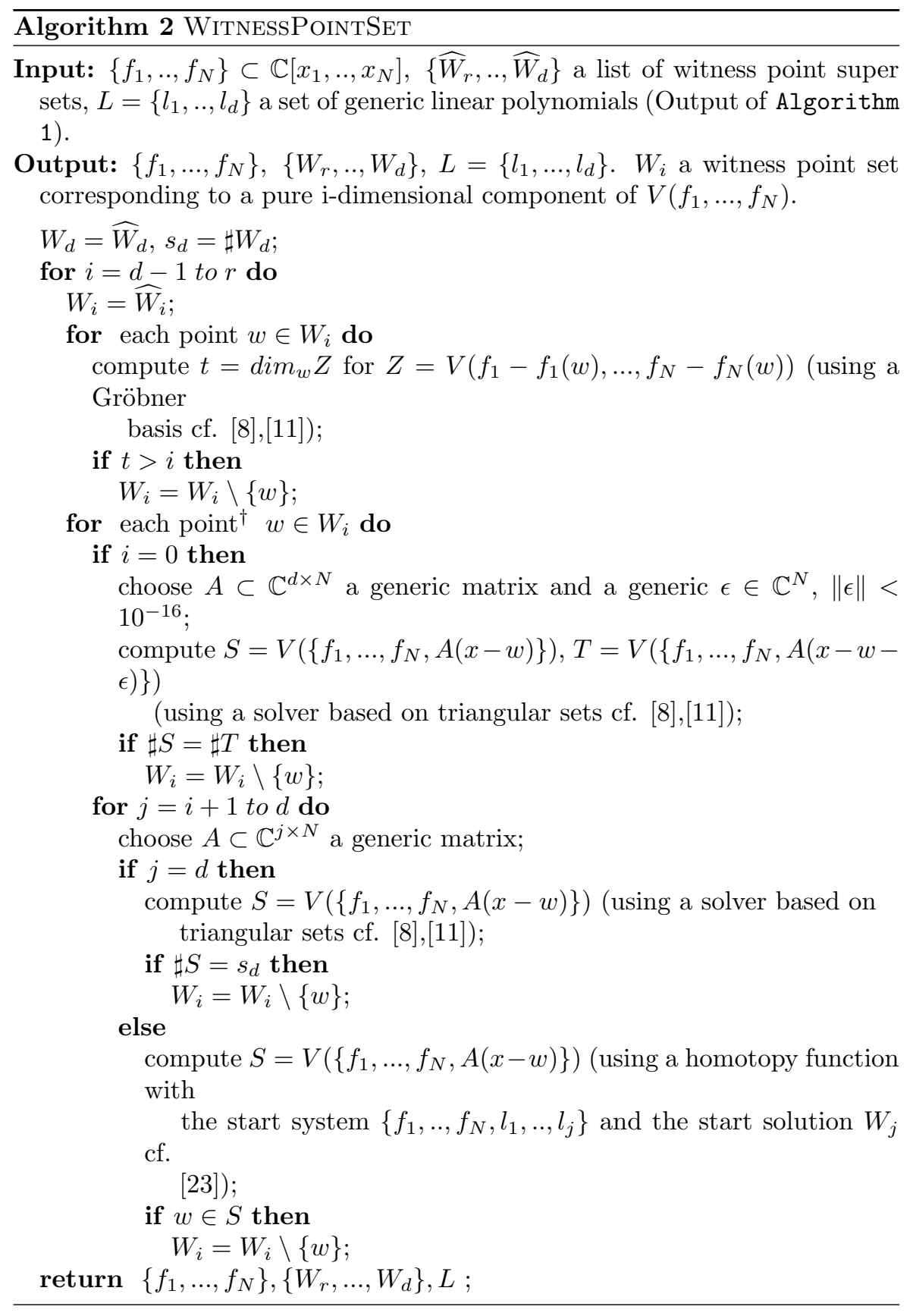




$$
Z_{\varphi\left(x, f_{1}(w), \ldots, f_{N}(w)\right)}=V\left(f_{1}-f_{1}(w), \ldots, f_{N}-f_{N}(w)\right) \subset \mathbb{C}^{N} .
$$

It follows (cf. [9], proposition 3.4)

$$
\left.t:=\operatorname{dim}_{w} V\left(f_{1}-f_{1}(w), \ldots, f_{N}-f_{N}(w)\right) \leq \operatorname{dim}_{v} V\left(f_{1}, \ldots, f_{N}\right)\right) .
$$

If $t>i$, then $w$ must be the approximate value of a point $v$ on a component of dimension greater then $i$. That means that $w \in J_{i}$.

If $i=0$, i.e. $w \in \widehat{W}_{0}$, then an $(N-d)$-dimensional generic linear space $V(A(x-w))$ meets the algebraic variety $V\left(f_{1}, \ldots, f_{N}\right)$ in a finite set $S$. If the $(N-d)$-dimensional generic linear space $V(A(x-w-\epsilon))$ passing through a neighborhood of $w$ meets $V\left(f_{1}, \ldots, f_{N}\right)$ in a set $T$ of the same cardinality, then there exists a neighborhood $U$ of $w$ such that $U \cap X \backslash\{w\} \neq \emptyset$. This implies that $w$ is not an isolated point in $V\left(f_{1}, \ldots, f_{N}\right)$, i.e. $w$ is on a component of positive dimension. This implies that $w \in J_{i}$. In case of $i>0$ the test whether $w$ is on a component of dimension $j \in\{d, d-1, \ldots, i+1\}$ is as follows. If $j=d$, the degree of the pure d-dimensional component is $s_{d}$. The d-dimensional generic linear space $V\left(A(x-w)^{T}\right)$ through $w$ meets $V\left(f_{1}, \ldots, f_{N}\right)$ in a finite set $S$ of cardinality greater or equal to $s_{d}$. If $\sharp S=s_{d}$, then $w$ is on the pure ddimensional component. It implies that $w \in J_{i}$. If $j<d$, we use the homotopy function to remove the junk points (cf. [23]).

\section{Partition Witness Point Sets}

In this section we show that the monodromy action on an algebraic variety $Z$ and the zero sum relation are sufficient to find the breakup of the k-witness point set $W_{k}$ into irreducible k-witness point sets. We present here a modified version of the algorithms described in ([14],[22]).

Let $Z$ be a pure k-dimensional algebraic variety in $\mathbb{C}^{N}$, and $Z=\cup_{i=1}^{r} Z_{i}$ be the irreducible decomposition of Z. Let $\pi: \mathbb{C}^{N} \longrightarrow \mathbb{C}^{k}$ be a generic projection and let $l \subset \mathbb{C}^{k}$ be a general line. Consider

- $\mathbb{W}_{l}:=\pi^{-1}(l) \cap Z$ a set of $\mathrm{r}$ different curves in $\mathbb{C}^{N}$.

- $U$ the non-empty open subset of $l$ consisting of all points $x \in l$ with $\pi^{-1}(x)$ transversal to $Z$.

- $W:=\pi^{-1}(x) \cap Z$ for a generic element $x \in U$, and $V$ a non-empty subset of W.

- $W_{i}:=\pi^{-1}(x) \cap Z_{i}$ for an irreducible k-dimensional component $Z_{i}$ of $\mathrm{Z}$.

- $\lambda: \mathbb{C}^{N} \longrightarrow \mathbb{C}$ a linear function, one-to-one on $W$. 
- For $y \in U$, let $V_{y}$ be a subset of $\pi^{-1}(y) \cap Z$ defined by

$$
V_{y}:=\left\{z \mid z \text { on a curve in } \mathbb{W}_{l} \text { through a point of } V\right\} \text {. }
$$

We define a function $s: U \longrightarrow \mathbb{C}$ by

$$
s(y)=\sum_{z \in V_{y}} \lambda(z)
$$

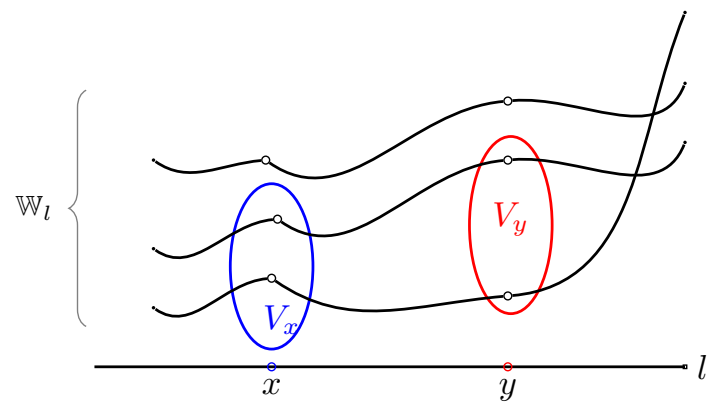

Theorem 4.1. Let $l, U, W, V, W_{i}$ for $i=1, \ldots, r$, and the functions $\lambda, s$ be as above.

If the function $s$ is continuous and $V \cap W_{i} \neq \emptyset$ for some $i \in\{1, \ldots, r\}$, then $W_{i} \subseteq V$.

Before proving the theorem we illustrate it by an example.

Example 4.1. Let $Z$ be the curve in $\mathbb{C}^{2}$ defined by the polynomial $f(x, y)=$ $\left(x^{2}+y^{2}-5\right)(x-2 y-3)$. Let $L_{1}$ be the line in $\mathbb{C}^{2}$ defined by the polynomial $l_{1}=x+y-3$. We define a homotopy function:

$$
\begin{gathered}
h(t, x(t), y(t)):=\left(\begin{array}{c}
\alpha(t) \\
f(x(t), y(t))
\end{array}\right) . \\
\alpha(t)=(1-t) l_{0}+t l_{1}=x+y-2 t-1, \text { where } l_{0}=x+y-1 .
\end{gathered}
$$

Then with conditions above $\alpha(t)$ maps a point in $L_{1} \cap Z$ to a point in $L_{0} \cap Z$ as $t$ goes from 1 to $0, L_{0}$ the line defined by $l_{0}$.

Proof (of Theorem 4.1). Assume that $W_{i} \nsubseteq V$. Since $W_{i} \cap V \neq \emptyset$, then there are $a, b \in W_{i}$ such that $a$ is not in $V$ and $b$ in $V$. Let $a_{1}, \ldots, a_{r}$ denote the points of the set $V \backslash\{b\}$. By Corollary 3.5 in [14] there is a loop $\alpha$ in the fundamental group $\pi_{1}\left(U, \pi^{-1}(x)\right)$ with $\alpha(0)=\alpha(1)$ which takes $a_{j}$ to $a_{j}$ for all $\mathrm{j}=1, \ldots, \mathrm{r}$, and interchanges $\mathrm{a}$ and $\mathrm{b}$. 
Since $\alpha$ is a continuous loop and $s: U \longrightarrow \mathbb{C}$ is continuous, the composition $s \circ \alpha:[0,1] \longrightarrow \mathbb{C}$ is continuous and

$$
\begin{aligned}
s(\alpha(1)) & =s(\alpha(0)) \\
\lambda(a)+\sum_{j=1}^{r} \lambda\left(a_{j}\right) & =\lambda(b)+\sum_{j=1}^{r} \lambda\left(a_{j}\right),
\end{aligned}
$$

as $t$ goes from 1 to 0 . This implies that $\lambda(a)=\lambda(b)$. But this contradicts the fact that $\lambda$ is one-to-one on $\mathrm{W}$. Thus $W_{i}$ is a subset of $V$.

Example 4.2. Let $Z$ be the curve in $\mathbb{C}^{2}$ defined be the polynomial $f(x, y)=$ $(y-x)(y-2 x)(y-3 x)$, and $Z=Z_{1} \cup Z_{2} \cup Z_{3}$ be the irreducible decomposition. Let $\pi: \mathbb{C}^{2} \longrightarrow \mathbb{C}$ be the projection given by $\pi(x, y)=x$, and $\lambda: \mathbb{C}^{2} \longrightarrow \mathbb{C}$, $\lambda(x, y)=y$.

Note that the restriction of $\pi$ to $Z, \pi_{Z}$ is proper and generically three-to-one with degree 3 equal to the degree of $Z$. $\lambda$ is one-to-one on the fiber $\pi^{-1}(y)=$ $\{(x, x),(x, 2 x),(x, 3 x)\}$. Let $L$ be the line defined by the linear polynomial $l(x, y)=x+y-2$. L intersects $Z$ in the finite set $W:=\left\{(1,1),\left(\frac{2}{3}, \frac{4}{3}\right),\left(\frac{1}{2}, \frac{3}{2}\right)\right\}$. Let $V:=\left\{(1,1),\left(\frac{2}{3}, \frac{4}{3}\right)\right\} \subset W$. The function $\sum_{v \in V} \lambda(v)$ given by $\lambda(x, x)+$ $\lambda(x, 2 x)=x+2 x=3 x$ is continuous. By the theorem above if an irreducible 1 -witness point set $W_{1}$ contains $\{(1,1)\}$ or $\left\{\left(\frac{2}{3}, \frac{4}{3}\right)\right\}$, then $W_{1}$ is a subset of $V$.

Now we will explain our modification of the algorithm to compute the irreducible witness point sets.

Let $Z_{k}=\cup_{i=1}^{r} Z_{k i}$ be the union of the irreducible k-dimensional components of the algebraic variety $Z=V\left(f_{1}, \ldots, f_{n}\right)$ and $L_{k}$ be the linear space in $\mathbb{C}^{N}$ defined by $k$ generic linear polynomials

$$
l_{j}=c_{j 0}+c_{j 1} x_{1}+\ldots+c_{j N} x_{N} .
$$

for $j=1, . ., k$ and $i=0,1, \ldots, N, c_{i j} \in \mathbb{C}$.

We use the generic linear space $L_{k}$ to define a projection $\pi: \mathbb{C}^{N} \longrightarrow \mathbb{C}^{k+1}, \pi\left(x_{1}, \ldots, x_{N}\right):=\left(z_{1}, \ldots, z_{k}, z_{k+1}\right)$ as follows:

$$
\left(\begin{array}{c}
x_{1} \\
\cdot \\
\cdot \\
\cdot \\
\cdot \\
x_{N}
\end{array}\right) \mapsto\left(\begin{array}{c}
z_{1} \\
\cdot \\
\cdot \\
\cdot \\
z_{k} \\
z_{k+1}
\end{array}\right):=\left(\begin{array}{ccccc}
c_{11} & c_{12} & \cdot & \cdot & c_{1 N} \\
c_{21} & c_{22} & \cdot & \cdot & c_{1 N} \\
\cdot & \cdot & \cdot & \cdot & \cdot \\
\cdot & \cdot & \cdot & \cdot & \cdot \\
c_{k 1} & c_{k 2} & \cdot & \cdot & c_{k N} \\
p_{1} & p_{2} & \cdot & \cdot & p_{N}
\end{array}\right) \cdot\left(\begin{array}{c}
x_{1} \\
\cdot \\
\cdot \\
\cdot \\
\cdot \\
x_{N}
\end{array}\right) \text {, }
$$

$p_{1}, \ldots, p_{N} \in \mathbb{C}$ randomly chosen.

Set $\lambda\left(x_{1}, \ldots, x_{N}\right):=z_{k+1}$ and $l:=V\left(z_{1}, \ldots, z_{k-1}\right) \subset \mathbb{C}^{k}$ the coordinate axis $z_{k}$ 
as in the theorem above. Let $L_{k, y}$ be the linear space defined by the linear polynomials $l_{1}, \ldots, l_{k-1}$ and $l_{k, y}:=y+c_{k 1} x_{1}+\ldots+c_{k N} x_{N}$. Let $W_{y}:=L_{k, y} \cap Z_{k}$ be the k-witness point set. For $y=c_{k 0}$, we fix a non-empty subset $V=V_{y} \subset$ $W_{y}$. In general let $V_{y}$ be the subset of $W_{y}$ consisting of all points which are on a curve in $\pi^{-1}(l) \cap Z_{k}$ through a point of $V$. To compute $V_{y}$ we use the homotopy function

$$
H(x(t), t)=(1-t) \cdot\left(\begin{array}{c}
l_{1} \\
\cdot \\
\cdot \\
l_{k} \\
f_{1} \\
\cdot \\
\cdot \\
f_{n}
\end{array}\right)+t .\left(\begin{array}{c}
l_{1} \\
\cdot \\
\cdot \\
l_{k}-c_{k 0}+y \\
f_{1} \\
\cdot \\
\cdot \\
f_{n}
\end{array}\right)
$$

as $t$ goes from 1 to 0 using $V$ as start system. Note that $\sharp V_{y}=\sharp V$.

Define the function $s: \mathbb{C} \longrightarrow \mathbb{C}$ by

$$
s(y):=\sum_{\left(x_{1}, \ldots, x_{N}\right) \in V_{y}} \lambda\left(x_{1}, \ldots, x_{N}\right) .
$$

To test the linearity of $s$, we take three values of y in $\mathbb{C}$, say $a, b, c$. If there exist $A, B \in \mathbb{C}$ such that

$$
(s(a)=A a+B, s(b)=A b+B) \Longrightarrow s(c)=A c+B,
$$

then $s$ is linear.

So far this is the approach which can be found in [14]. We now explain a modification.

The condition (4.1) of the linearity above is equivalent to the following equation

$$
s(a)(b-c)+s(b)(c-a)+s(c)(a-b)=0 .
$$

If $W_{k j} \cap V_{a} \neq \emptyset$ for some $j \in\{1, \ldots, r\}$ and the condition (4.1) is true, then $W_{k j} \subseteq V_{a}$ (Theorem 4.1). Let

$$
Z(y):=\left\{z=\sum_{t=1}^{N} p_{t} v_{t} \mid v=\left(v_{1}, \ldots, v_{N}\right) \in V_{y}, p=\left(p_{1}, \ldots, p_{N}\right) \in \mathbb{C}^{N}\right\} .
$$

Then

$$
s(y)=\sum_{v \in V_{y}} \lambda(v)=\sum_{v \in V_{y}}\left(\sum_{t=1}^{N} p_{t} v_{t}\right)=\sum_{z \in Z(y)} z .
$$


The continuation of the homotopy function implies that the i-th points in the sets $V_{a}, V_{b}$ and $V_{c}$ are on the same irreducible component. Let $V_{a}:=$ $\left\{v_{1}, \ldots, v_{m}\right\}, V_{b}:=\left\{\bar{v}_{1}, \ldots, \bar{v}_{m}\right\}$ and $V_{c}:=\left\{\hat{v}_{1}, \ldots, \hat{v}_{m}\right\}$ be the sets computed by using the homotopy function above . Let $Z(a):=\left\{a_{1}, \ldots, a_{m}\right\}, Z(b):=$ $\left\{b_{1}, \ldots, b_{m}\right\}$ and $Z(c):=\left\{c_{1}, \ldots, c_{m}\right\}$ be the sets corresponding to the set $V_{a}$, $V_{b}$ and $V_{c}$ respectively.

From (4.2) we obtain an equivalent condition to (4.1)

$$
(b-c) \sum_{i=1}^{m} a_{i}+(c-a) \sum_{i=1}^{m} b_{i}+(a-b) \sum_{i=1}^{m} c_{i}=0
$$

The condition (4.3) is called zero sum relation (cf. [7]) of a given subset $V_{a} \subseteq W$ denoted by $Z S R\left(V_{a}\right)$. The sets $V_{a}, V_{b}$ and $V_{c}$ have distinct points and the same cardinality $m$, then obviously

$$
Z S R\left(V_{a}\right)=\sum_{a_{i} \in V_{a}} Z S R\left(\left\{a_{i}\right\}\right)
$$

where $Z S R\left(\left\{a_{i}\right\}\right)=(b-c) a_{i}+(c-a) b_{i}+(a-b) c_{i}$ is defined as the zero sum relation of a given point in $V_{a}$.

The following algorithm computes irreducible witness point sets. The correctness of the Algorithm 3 follows from the Theorem (4.1).

We give an example of a pure 2-dimensional variety $Z$ which is a union of two 2-dimensional irreducible components $Z_{1}$ and $Z_{2}$. $Z_{1}$ is of degree three and $Z_{2}$ is of degree two. The 2 -witness point set $W$ for $Z$ is given as a finite subset of $Z$ consisting of five points $\left\{w_{1}, w_{2}, w_{3}, w_{4}, w_{5}\right\} . Z_{1}$ should contain three points $W_{1}:=\left\{w_{1}, w_{2}, w_{3}\right\}$ and the remaining points $W_{2}:=\left\{w_{4}, w_{5}\right\}$ are on $Z_{2}$. The algorithms (cf. [14],[22]) use the homotopy function at least nine times to breakup $\mathrm{W}$ into $W_{1}$ and $W_{2}$. We will show below that we do not need more than five times to use the homotopy function to breakup $\mathrm{W}$ into $W_{1}$ and $W_{2}$.

\section{Example 4.3. .}

Let $Z$ be the algebraic variety of dimension two in $\mathbb{C}^{3}$ defined by the polynomial $f(x, y, z)=\left(x^{3}+z\right)\left(x^{2}-y\right)$. Let $L$ be the linear space of dimension one in $\mathbb{C}^{3}$ defined by the linear equations $l_{1}=4 x+7 y+2 z+6, l_{2}=5 x+7 y+3 z+6$. Then $W:=L \cap Z=\left\{w_{1}, w_{2}, w_{3}, w_{4}, w_{5}\right\}$, where $w_{1}=(1,-1.1428571429,-1), w_{2}=$ $(0,-0.8571428571,0)$,

\footnotetext{
${ }^{\ddagger}$ the $i-t h$ point in $\mathrm{R}$ corresponds to the $i-t h$ point in $W_{a}$;

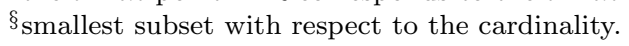




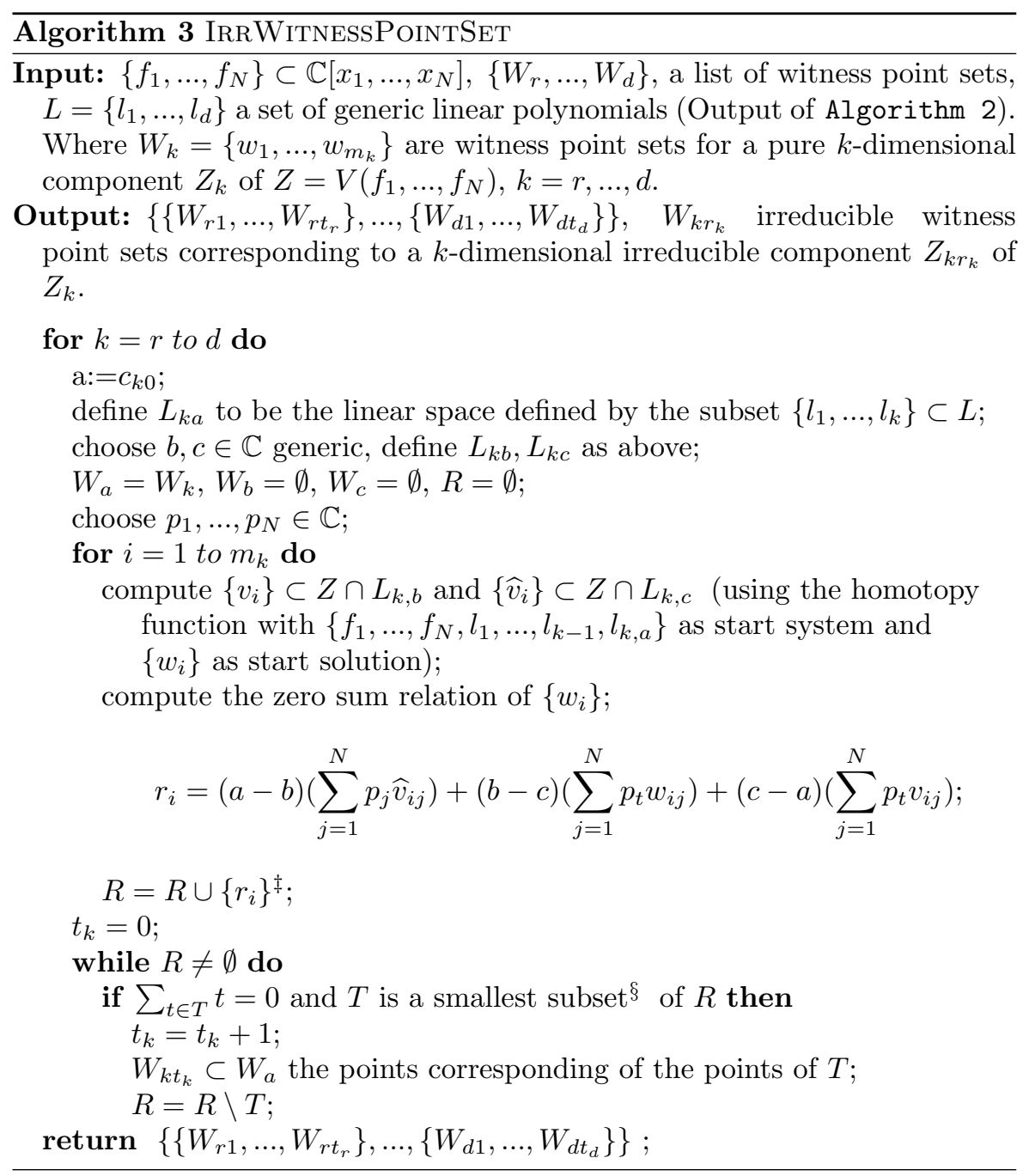






We now illustrate the Algorithm 3:

- Use the linear space $L_{1}$ to define the linear projection $\pi: \mathbb{C}^{3} \longrightarrow \mathbb{C}^{3}$ as follows

$$
\pi(x, y, z):=\left(\begin{array}{ccc}
4 & 7 & 2 \\
5 & 7 & 3 \\
1 & 2 & 3
\end{array}\right)\left(\begin{array}{l}
x \\
y \\
z
\end{array}\right)=(4 x+7 y+2 z, 5 x+7 y+3 z, x+2 y+3 z)
$$

- Define the linear space $L_{1, c}$ of dimension one in $\mathbb{C}^{3}$ by the linear equations $l_{1}=4 x+7 y+2 z+6, l_{c}=5 x+7 y+3 z+c$, where $c$ is generically chosen in $\mathbb{C}$. Then

$$
\pi_{Z \cap L_{1, c}}(x, y)=(-6,-c, x+2 y+3 z)
$$

- Define the linear function $\lambda: \mathbb{C}^{2} \longrightarrow \mathbb{C}$ by $\lambda(x, y, z):=x+2 y+3 z$.

- For $a=6$, let $V_{1}=V_{a}:=\left\{w_{11}=(1,-1.1428571429,-1)\right\} \subset W, L_{1, a}:=$ $L$ the linear space defined by $l_{1}=4 x+7 y+2 z+6, l_{a}=5 x+7 y+$ $3 z+6$. Then $Z(a)=\left\{\sum_{v \in V_{a}} \lambda(v)=w_{11}[1]+2\left(w_{11}[2]\right)+3\left(w_{11}[3]\right)\right\}=$ $\{-4.2857142858\}$.

- Let $b=9, L_{1, b}$ the linear space defined by $l_{1}=4 x+7 y+2 z+6, l_{b}=$ $5 x+7 y+3 z+9$. Compute $V_{b}:=\left(t L_{1, a}+(1-t) L_{1, b}\right) \cap Z=\left\{w_{12}=\right.$ $(1.671699881657157,-0.4776285376163331,-4.671699881657164)\}$ as $t$ goes from 1 to 0 , using $V_{a}$ as a start solution. $Z(b)=\left\{w_{12}[1]+\right.$ $\left.2\left(w_{12}[2]\right)+3\left(w_{12}[3]\right)\right\}=\{-13.2986568385470012\}$.

- Let $c=63, L_{1, c}$ the linear space defined by $l_{1}=4 x+7 y+2 z+$ $6, l_{c}=5 x+7 y+3 z+63$. Compute $V_{c}:=\left(t L_{1, a}+(1-t) L_{1, c}\right) \cap Z=$ $\left\{w_{13}=(3.935100643260828,14.30425695906836,-60.93510064326094)\right\}$ as $t$ goes

from 1 to 0 , using $V_{a}$ as a start solution. $Z(c)=\left\{w_{13}[1]+2\left(w_{13}[2]\right)+\right.$ $\left.3\left(w_{13}[3]\right)\right\}=\{-150.261687368385272\}$.

ף we use the notation $w_{i j}=\left(w_{i j}[1], w_{i j}[2], w_{i j}[3]\right)$ for $\mathrm{i}=1, . ., 5, \mathrm{j}=1,2,3$. 
zero sum relation of $V_{1}=\{(1,-1.1428571429,-1)\}$ :

$$
\begin{gathered}
r_{1}:=\sum_{a \in Z(a)}(b-c)+\sum_{b \in Z(b)}(c-a)+\sum_{c \in Z(c)}(a-b)= \\
=-75.8098062588232524 .
\end{gathered}
$$

The zero sum relation set of $V_{1}=\{(1,-1.1428571429,-1)\}$ is $R_{1}:=\left\{r_{1}=\right.$ $-75.8098062588232524\}$.

- Let $a=6, V_{a}:=\left\{w_{11}=(0,-0.8571428571,0)\right\} \subset W, L_{1, a}:=L$ the linear space defined by $l_{1}=4 x+7 y+2 z+6, l_{a}=5 x+7 y+3 z+$ 6. Then $Z(a)=\left\{\sum_{v \in V_{a}} \lambda(v)=w_{11}[1]+2\left(w_{11}[2]\right)+3\left(w_{11}[3]\right)\right\}=$ $\{-1.7142857142\}$.

- Let $b=9, L_{1, b}$ the linear space defined by $l_{1}=4 x+7 y+2 z+6, l_{b}=$ $5 x+7 y+3 z+9$. Compute $V_{b}:=\left(t L_{1, a}+(1-t) L_{1, b}\right) \cap Z=\left\{w_{12}=\right.$ $(-0.8358499408285809+i * 1.046869318849985,0.2388142688081706-$ $i * 0.2991055196714253,-2.164150059171436-i * 1.046869318849981)\}$ as $t$ goes from 1 to 0 , using $V_{a}$ as a start solution. $Z(b)=\left\{w_{12}[1]+\right.$ $\left.2\left(w_{12}[2]\right)+3\left(w_{12}[3]\right)\right\}=\{-6.8506715807265477-i * 2.6919496770428086\}$.

- Let $c=63, L_{1, c}$ the linear space defined by $l_{1}=4 x+7 y+2 z+6, l_{c}=$ $5 x+7 y+3 z+63$. Compute $V_{c}:=\left(t L_{1, a}+(1-t) L_{1, c}\right) \cap Z=$ $\left\{w_{13}=(-1.967550321630417+i * 3.257877039491183\right.$, $15.99072866332302-i * 0.9308220112831772$, $-55.03244967836969-i * 3.257877039491242) ;\}$ as $t$ goes from 1 to 0 , using $V_{a}$ as a start solution. $Z(c)=\left\{w_{13}[1]+2\left(w_{13}[2]\right)+3\left(w_{13}[3]\right)\right\}=$ $\{-135.083442030093447-i * 8.3773981015488974\}$.

zero sum relation of $V_{2}=\{(0,-0.8571428571,0)\}$ :

$$
\begin{aligned}
& r_{2}:=\sum_{a \in Z(a)}(b-c)+\sum_{b \in Z(b)}(c-a)+\sum_{c \in Z(c)}(a-b)= \\
& =107.3334745556671221-i * 128.308937286793398 .
\end{aligned}
$$

The zero sum relation set of $V_{2}=\{(0,-0.8571428571,0)\}$ is $R_{2}:=\left\{r_{2}=107.3334745556671221-i * 128.308937286793398\right\}$.

- For the other points $V_{3}=\left\{w_{3}\right\}, V_{4}=\left\{w_{4}\right\}$ and $V_{5}=\left\{w_{5}\right\}$, we found the zero sum relations:

$R_{3}:=\left\{r_{3}=-9.38237104997583366+i * 127.0170767088\right\}$,

$R_{4}:=\left\{r_{4}=-31.5236682999307779+i * 128.3089372867945956\right\}$ and $R_{5}:=\left\{r_{5}=9.382371038077068-i * 127.0170767088\right\}$. 
- The set of zero sum relation for all points of $W$ is $R=\cup_{j=1}^{5} R_{j}=$ $\left\{r_{1}, r_{2}, r_{3}\right.$,

$\left.r_{4}, r_{5}\right\}$, where $i$-th point in $W$ corresponds $i$-th point in $R$.

- Find the smallest subset $T$ of $R$ with $\sum_{t \in T} t=0$, which corresponds an irreducible witness point set of $W$. Then we get $T_{1}=\left\{r_{3}, r_{5}\right\}$, $T_{2}=\left\{r_{1}, r_{2}, r_{4}\right\}$ corresponding to the irreducible witness point sets $W_{1}=$ $\left\{w_{3}, w_{5}\right\}, W_{2}=\left\{w_{1}, w_{2}, w_{4}\right\}$ respectively.

Remark 4.1. The points of a witness point set are computed approximately by using the homotopy continuation method. Therefore the result of the zero sum relation is only almost zero.

\section{Examples and timings with Singular and Bertini}

In this section we provide examples with timings of the algorithms WitnessPointSuperSet, WitnessPointSet, and IrrWitnessPointSet implemented in Singular to computell the numerical decomposition of a given algebraic variety defined by a polynomial system and compare them with the results of BERTINI . Each step of the numerical decomposition is parallelizable. For our comparisons we did not use the parallel version of BERTINI .

We tested two versions of the implementation in BERTINI using the cascade algorithm (cf. [15]) and using the regenerative cascade (cf. [16]) algorithm. For the timings we used the 32-bit version of Singular 3-1-1 (cf. [8]) and Bertini 1.2 (cf. [3]) on an Intel@ Core(TM)2 Duo CPU P8400 @ $2.26 \mathrm{GHz}$ $2.27 \mathrm{GHz}, 4$ GB RAM under the Kubuntu Linux operating system.

Let $\mathrm{Z}$ be the algebraic variety defined by the following polynomial system:

Example 5.1. (cf. [18]).

$$
f(x, y, z)=\left(\begin{array}{c}
\left(y-x^{2}\right)\left(x^{2}+y^{2}+z^{2}-1\right)\left(x-\frac{1}{2}\right) \\
\left(z-x^{3}\right)\left(x^{2}+y^{2}+z^{2}-1\right)\left(y-\frac{1}{2}\right) \\
\left(y-x^{2}\right)\left(z-x^{3}\right)\left(x^{2}+y^{2}+z^{2}-1\right)\left(z-\frac{1}{2}\right)
\end{array}\right)
$$

Example 5.2. (cf. [23], Example 13.6.4).

$$
f(x, y, z)=\left(\begin{array}{c}
x\left(y^{2}-x^{3}\right)(x-1) \\
x\left(y^{2}-x^{3}\right)(y-2)(3 x+y)
\end{array}\right)
$$

\footnotetext{
$\|$ The Singular implementation uses BERTINI to compute the solutions of the homotopy function.
} 
Example 5.3.

$$
f(x, y, z)=\left(\begin{array}{c}
\left(x^{3}+z\right)\left(x^{2}-y\right) \\
\left(x^{3}+y\right)\left(x^{2}-z\right) \\
\left(x^{3}+z\right)\left(x^{3}+y\right)\left(z^{2}-y\right)
\end{array}\right)
$$

Example 5.4.

$$
f(x, y, z)=\left(\begin{array}{c}
x\left(y^{2}-x^{3}\right)(x-1) \\
x(3 x+y)\left(y^{2}-x^{3}\right)(y-2) \\
x\left(y^{2}-x^{3}\right)\left(x^{2}-y\right)
\end{array}\right)
$$

Example 5.5.

$$
f(x, y, z)=\left(\begin{array}{c}
(x-1)\left(\left(x^{3}+z\right)+\left(x^{2}-y\right)\right) \\
\left(x^{3}+z\right)\left(x^{2}-y\right) \\
\left(x^{3}+z\right)\left(x^{2}-1\right)
\end{array}\right)
$$

Example 5.6.

$$
f(x, y, z)=\left(\begin{array}{c}
\left(y-x^{2}\right)\left(x^{2}+y^{2}+z^{2}-1\right)\left(x-\frac{1}{2}\right)+x^{5} \\
\left(z-x^{3}\right)\left(x^{2}+y^{2}+z^{2}-1\right)\left(y-\frac{1}{2}\right)+y^{4} \\
\left(y-x^{2}\right)\left(z-x^{3}\right)\left(x^{2}+y^{2}+z^{2}-1\right)\left(z-\frac{1}{2}\right)+z^{6}
\end{array}\right)
$$

Example 5.7.

$$
f(x, y, z)=\left(\begin{array}{c}
x\left(y^{2}-x^{3}\right)(x-1)+y^{2} \\
x\left(y^{2}-x^{3}\right)(y-2)(3 x+y)+x^{3}
\end{array}\right)
$$

Example 5.8.

$$
f(x, y, z)=\left(\begin{array}{c}
\left(x^{3}+z\right)\left(x^{2}-y\right)+x^{4} \\
\left(x^{3}+y\right)\left(x^{2}-z\right)+y^{3} \\
\left(x^{3}+z\right)\left(x^{3}+y\right)\left(z^{2}-y\right)+z^{5}
\end{array}\right)
$$

Example 5.9.

$$
f(x, y)=\left(\begin{array}{c}
-3568891411860300072 x^{5}+1948764938 x^{4}+ \\
3568891411860300072 x^{2} y^{2}-1948764938 x y^{2} \\
-5105200242937540320 x^{5} y-1701733414312513440 x^{4} y^{2}+ \\
11692589628 x^{5}+3897529876 x^{4} y+5105200242937540320 x^{2} y^{3}+ \\
1701733414312513440 x y^{4}-11692589628 x^{2} y^{2}-3897529876 x y^{3}
\end{array}\right)
$$




\section{Example 5.10.}

$$
f(x, y, z)=\left(\begin{array}{c}
-356737285367005125 x^{5}-92300457164036000 x^{3} y+ \\
1121648050080163317 x^{2} z+290209720279281056 y z \\
-356737285367005125 x^{5}+887060318883271500 x^{3} z+ \\
1121648050080163317 x^{2} y-2789081819567309964 y z \\
\\
-356737285367005125 x^{5} z^{2}+356737285367005125 x^{5} y+ \\
887060318883271500 x^{3} z^{3}-887060318883271500 x^{3} y z+ \\
1121648050080163317 x^{2} z^{3}-1121648050080163317 x^{2} y z- \\
2789081819567309964 z^{4}+2789081819567309964 y z^{2}
\end{array}\right)
$$

Example 5.11.

$$
f(x, y, z)=\left(\begin{array}{c}
x^{5} y^{2}+2 x^{3} y^{4}+x y^{6}+2 x^{3} y^{2} z^{2}+2 x y^{4} z^{2}+x y^{2} z^{4}-x^{4} y^{2} \\
-2 x^{2} y^{4}-y^{6}-x^{5} z-2 x^{3} y^{2} z-x y^{4} z-2 x^{2} y^{2} z^{2}-2 y^{4} z^{2}- \\
2 x^{3} z^{3}-2 x y^{2} z^{3}-y^{2} z^{4}-x z^{5}-3 x^{3} y^{2}-3 x y^{4}+x^{4} z+ \\
2 x^{2} y^{2} z+y^{4} z-3 x y^{2} z^{2}+2 x^{2} z^{3}+2 y^{2} z^{3}+z^{5}+3 x^{2} y^{2}+ \\
3 y^{4}+3 x^{3} z+3 x y^{2} z+3 y^{2} z^{2}+3 x z^{3}+2 x y^{2}-3 x^{2} z-3 y^{2} z- \\
3 z^{3}-2 y^{2}-2 x z+2 z \\
\\
x^{6} y+2 x^{4} y^{3}+x^{2} y^{5}+2 x^{4} y z^{2}+2 x^{2} y^{3} z^{2}+x^{2} y z^{4}- \\
5 x^{6}-10 x^{4} y^{2}-5 x^{2} y^{4}-x^{4} y z-2 x^{2} y^{3} z-y^{5} z-10 x^{4} z^{2}- \\
10 x^{2} y^{2} z^{2}-2 x^{2} y z^{3}-2 y^{3} z^{3}-5 x^{2} z^{4}-y z^{5}-3 x^{4} y- \\
3 x^{2} y^{3}+5 x^{4} z+10 x^{2} y^{2} z+5 y^{4} z-3 x^{2} y z^{2}+10 x^{2} z^{3}+ \\
10 y^{2} z^{3}+5 z^{5}+15 x^{4}+15 x^{2} y^{2}+3 x^{2} y z+3 y^{3} z+15 x^{2} z^{2} \\
+3 y z^{3}+2 x^{2} y-15 x^{2} z-15 y^{2} z-15 z^{3}-10 x^{2}-2 y z+10 z \\
\\
x^{6} y^{2} z+2 x^{4} y^{4} z+x^{2} y^{6} z+2 x^{4} y^{2} z^{3}+2 x^{2} y^{4} z^{3}+ \\
x^{2} y^{2} z^{5}-7 x^{6} y^{2}-14 x^{4} y^{4}-7 x^{2} y^{6}-x^{6} z^{2}-17 x^{4} y^{2} z^{2}- \\
17 x^{2} y^{4} z^{2}-y^{6} z^{2}-2 x^{4} z^{4}-11 x^{2} y^{2} z^{4}-2 y^{4} z^{4}-x^{2} z^{6}- \\
y^{2} z^{6}+7 x^{6} z+18 x^{4} y^{2} z+18 x^{2} y^{4} z+7 y^{6} z+15 x^{4} z^{3}+ \\
27 x^{2} y^{2} z^{3}+15 y^{4} z^{3}+9 x^{2} z^{5}+9 y^{2} z^{5}+z^{7}+21 x^{4} y^{2}+ \\
21 x^{2} y^{4}-4 x^{4} z^{2}+13 x^{2} y^{2} z^{2}-4 y^{4} z^{2}-11 x^{2} z^{4}-11 y^{2} z^{4}- \\
7 z^{6}-21 x^{4} z-40 x^{2} y^{2} z-21 y^{4} z-24 x^{2} z^{3}-24 y^{2} z^{3}-3 z^{5}- \\
14 x^{2} y^{2}+19 x^{2} z^{2}+19 y^{2} z^{2}+21 z^{4}+14 x^{2} z+14 y^{2} z+ \\
2 z^{3}-14 z^{2}
\end{array}\right.
$$


Example 5.12.

$$
f\left(x_{1}, x_{2}, x_{3}, x_{4}, x_{5}\right)=\left(\begin{array}{c}
x_{5}^{2}+x_{1}+x_{2}+x_{3}+x_{4}-x_{5}-4 \\
x_{4}^{2}+x_{1}+x_{2}+x_{3}-x_{4}+x_{5}-4 \\
x_{3}^{2}+x_{1}+x_{2}-x_{3}+x_{4}+x_{5}-4 \\
x_{2}^{2}+x_{1}-x_{2}+x_{3}+x_{4}+x_{5}-4 \\
x_{1}^{2}-x_{1}+x_{2}+x_{3}+x_{4}+x_{5}-4
\end{array}\right)
$$

Example 5.13.

$$
f(a, b, c, d, e, f, g)=\left(\begin{array}{c}
a^{2}+2 d e+2 c f+2 b g+a \\
2 a b+e^{2}+2 d f+2 c g+b \\
b^{2}+2 a c+2 e f+2 d g+c \\
2 b c+2 a d+f^{2}+2 e g+d \\
c^{2}+2 b d+2 a e+2 f g+e \\
2 c d+2 b e+2 a f+g^{2}+f \\
d^{2}+2 c e+2 b f+2 a g+g
\end{array}\right)
$$

Example 5.14. cyclic 4-roots problem.(cf.[5],[6]).

Example 5.15. cyclic 5-roots problem.(cf.[5],[6]).

Example 5.16. cyclic 6-roots problem.(cf.[5],[6]).

Example 5.17. cyclic 7-roots problem.(cf.[5],[6]).

Example 5.18. cyclic 8-roots problem.(cf.[5],[6]).

Example 5.19.

$$
\begin{gathered}
f\left(x_{11}, x_{12}, x_{13}, x_{14}, x_{15}, x_{21}, x_{22}, x_{23}, x_{24}, x_{25}, x_{31}, x_{32}, x_{33}, x_{34}, x_{35}\right)= \\
=\left(\begin{array}{l}
-x_{12} x_{21}+x_{11} x_{22} \\
-x_{13} x_{22}+x_{12} x_{23} \\
-x_{14} x_{23}+x_{13} x_{24} \\
-x_{15} x_{24}+x_{14} x_{25} \\
-x_{22} x_{31}+x_{21} x_{32} \\
-x_{23} x_{32}+x_{22} x_{33} \\
-x_{24} x_{33}+x_{23} x_{34} \\
-x_{25} x_{34}+x_{24} x_{35}
\end{array}\right)
\end{gathered}
$$

Table 1 summarizes the results of the timings to compute the numerical decomposition**. 
Remark 5.1. The timings in the following table show that for an increasing number of variables the original method of (cf.[14],[15],[18],[22],[23]) becomes more efficient. One reason is that the computation of triangular sets which is used in SINGULAR for solving polynomial systems is expensive in this case. Therefore the Algorithm 1, Algorithm 2 become slow in this situation. This is not true for the Algorithm 3.

Replacing the solving of polynomial systems using triangular sets by homotopy function methods but keeping the computation of the dimension and starting at this dimension is more efficient also in case of a large number of variables.

\begin{tabular}{|r|r|r|r|}
\hline Example & BERTINI & BERTINI $(\mathrm{re})$ & SINGULAR \\
\hline \hline 5.1 & $134.45 \mathrm{~s}$ & $39 \mathrm{~s}$ & 36.07 \\
\hline 5.2 & $3.08 \mathrm{~s}$ & $2.5 \mathrm{~s}$ & $1.49 \mathrm{~s}$ \\
\hline 5.3 & $1 \mathrm{~min} 21.28 \mathrm{~s}$ & $27.4 \mathrm{~s}$ & $4.02 \mathrm{~s}$ \\
\hline 5.4 & $18.56 \mathrm{~s}$ & $2.7 \mathrm{~s}$ & $1.77 \mathrm{~s}$ \\
\hline 5.5 & $15.36 \mathrm{~s}$ & $8.6 \mathrm{~s}$ & $1.29 \mathrm{~s}$ \\
\hline 5.6 & $4 \min 13 \mathrm{~s}$ & $15 \min 2 \mathrm{~s}$ & 2 min $27 \mathrm{~s}$ \\
\hline 5.7 & $1.83 \mathrm{~s}$ & $1.6 \mathrm{~s}$ & $0.39 \mathrm{~s}$ \\
\hline 5.8 & $3 \min 29 \mathrm{~s}$ & $10 \min 43 \mathrm{~s}$ & $1.69 \mathrm{~s}$ \\
\hline 5.9 & $16 \mathrm{~s}$ & $7 \mathrm{~s}$ & $2 \mathrm{~s}$ \\
\hline 5.10 & $2 \min 57 \mathrm{~s}$ & $28 \mathrm{~s}$ & $2 \min 35 \mathrm{~s}$ \\
\hline 5.11 & $44 \min 56 \mathrm{~s}$ & $2 \min 37 \mathrm{~s}$ & $4 m i n 3 \mathrm{~s}$ \\
\hline 5.12 & $4.73 \mathrm{~s}$ & $6 \mathrm{~s}$ & $0.37 \mathrm{~s}$ \\
\hline 5.13 & $5.84 \mathrm{~s}$ & $8 \mathrm{~s}$ & $1 \mathrm{~s}$ \\
\hline 5.14 & $1.43 \mathrm{~s}$ & $4.3 \mathrm{~s}$ & $0.79 \mathrm{~s}$ \\
\hline 5.15 & $3.54 \mathrm{~s}$ & $10 \mathrm{~s}$ & $0.57 \mathrm{~s}$ \\
\hline 5.16 & $3 \mathrm{~min} 23.26 \mathrm{~s}$ & 2 min $29 \mathrm{~s}$ & $1.43 \mathrm{~s}$ \\
\hline 5.17 & $2 \mathrm{~h} 11 \mathrm{~min} 57 \mathrm{~s}$ & $32 \mathrm{~min} 17 \mathrm{~s}$ & stopped after $5 \mathrm{~h}$ \\
\hline 5.18 & $19 \mathrm{~h} 48 \mathrm{~min} 17 \mathrm{~s}$ & $6 \mathrm{~h} 45 \mathrm{~min} 2 \mathrm{~s}$ & stopped after $50 \mathrm{~h}$ \\
\hline 5.19 & $1 \mathrm{~min} 57 \mathrm{~s}$ & $51 \mathrm{~s}$ & stopped after $3 \mathrm{~h}$ \\
\hline
\end{tabular}

Table 1: Total running times for computing a numerical decomposition of the examples above

**(re) means using the regenerative cascade algorithm instead of the cascade algorithm 


\section{References}

[1] E. L. Allgower and K. Georg. Numerical Continuation Methods, an Introduction, Springer Series in Comput. Math, Vol. 13, Springer- Veralg, Berlin, Heidelberg, New Yourk, 1990.

[2] E. Arbarello, M. Coranalba, P.A. Griffths and J. Harris. Geometry of Algebraic Curves, volume I. Volume 267 of Grundlehren Math. Wiss., Springer Verlag, New York, 1985.

[3] D. J. Bates, J. D. Hauenstein, A. J. Sommese and C. W. Wampler. BERTINI : Software for Numerical Algebraic Geometry. http://www.nd.edu/ sommese/bertini/.

[4] S. C. Billups, A. P. Morgan and L. T. Watson . Algorithm 652. Hompack: A Suite codes for Globally Convergent Homotopy Algorithms. ACM Transactions on Mathematical Software, Vol.13. No. 3, Septemper 1987, Pages 281- 310.

[5] G. Björck and R. Fröberg. A faster way to count the solutions of inhomogeneous systems of algebraic equations, with applications to cyclic n-roots. J. Symbolic Computation, 12:329- 336, 1991.

[6] G. Björck. Functions of modulus one on Zn whose Fourier transforms have constant modulus, and cyclic n-roots. In: J. S. Byrnes and J. F. Byrnes, Editors, Recent Advances in Fourier Analysis and its Applications 315, NATO Adv. Sci. Inst. Ser. C. Math. Phys. Sci., Kluwer (1989), pp. 131-140.

[7] R. M. Corless, A. Galligo, I. S. Kotsireas and S. M. Watt. A GeometricNumeric Algorithm for Absolute Factorization of Multivariate Polynomials. ISSAC 2002, July 7- 10, 2002, Lille, France.

[8] W. Decker, G.-M. Greuel, G. Pfister and H. Schönemann. SinguLAR 3-1-1 - A computer algebra system for polynomial computations. http://www.singular.uni-kl.de (2010).

[9] G. Fischer. Complex Alalytic Geometry, Lecture Notes in Mathematics 538 (1976).

[10] W. Fulton. Intersection Theory, volume (3) 2 of Ergeb. Math. Grenzgeb. Springer Verlag, Berlin, 1984.

[11] G.-M. Greuel and G. Pfister. A Singular Introduction to Commutative Algebra. Second edition, Springer (2007). 
[12] A. P. Morgan and L. T. Watson. A globally convergent parallel algorithm for zeros of polynomial systems. Nonlinear Anal. (1989), 13(11), 13391350 .

[13] A. P. Morgan and A. J. Sommese. Computing all solutions to polynomial systems using homotopy continuation. Appl. Math. Comput., 115- 138. Errata: Appl. Math. Comput. 51 (1992), p. 209. Nonlinear Anal.

[14] A. J. Sommese, J. Verschelde and C. W. Wampler. Symmetric functions applied to decomposing solution sets of polynomial systems. Vol. 40, No. 6, pp. 2026-2046. 2002 Society for Industrial and Applied Mathematics.

[15] A. J. Sommese and J. Verschelde. Numerical homotopies to compute generic points on positive dimensional algebraic sets. J. of Complexity 16(3):572- 602, (2000).

[16] A. J. Sommese, C. W. Wampler and J. D. Hauenstein. Regenerative cascade homotopies for solving polynomial systems. Applied Mathematics and Computation 218(4): 1240-1246 (2011)

[17] A. J. Sommese, J. Verschelde and C. W. Wampler. (2002a). A method for tracking singular paths with application to the numerical decomposition . In algebraic geometry (pp. 329- 345). Berlin: de Gruyter.

[18] A. J. Sommese, J. Verschelde and C. W. Wampler. (2001a). Numerical decomposition of solution sets of polynomial systems into irreducible components. SIAM J. Number. Anal., 38(6), 2022-2046. NID

[19] A. J. Sommese, J. Verschelde and C. W. Wampler. (2003). Numerical irreducible decomposition using PHCpack. In algebra, geometry, and software systems (pp. 109- 129). Berlin: Springer.

[20] A. J. Sommese, J. Verschelde and C.W. Wampler. Numerical decomposition of the solution sets of polynomial systems into irreducible components. SIAM J. Numer. Anal. 38(6):2022- 2046, 2001.

[21] A. J. Sommese, J. Verschelde and C. W. Wampler. Numerical irreducible decomposition using projections from points on components. In Symbolic Computation: Solving Equations in Algebra, Geometry, and Engineering, volume 286 of contemporary Mathematics, edited by E. L. Green, S. Hosten, R. C. Laubenbacher and V. Powers, pages 27- 51. AMS 2001. 
[22] A. J. Sommese, J. Verschelde and C. W. Wampler. Using monodromy to decompose solution sets of polynomial systems into irreducible components. In Application of Algebraic Geometry to Coding Theory, Physics and Computation, edited by C. Ciliberto , F. Hirzebruch, R. Miranda and M. Teicher. Proceedings of a NATO Conference, February 25- March 1, 2001, Eilat, Israel. Pages 297- 315, Kluwer Academic Publishers.

[23] A. J. Sommese and C. W. Wampler. The Numerical Solution of Systems of Polynomials Arising in Engineering and Science. ISBN 981- 256- 1846. Word Scientific Publishing Co. Plte. Ltd. 2005.

[24] A. J. Sommese and C. W. Wampler. Numerical algebraic geometry. In the mathematics of numerical analysis. (Park City, UT, 1995), Vol. 32 of lectures in Appl. Math. (pp. 749- 763). Providence, RI: Amer. Math. Soc.

[25] J. Verschelde(1996). Homotopy continuation methods for solving polynomial systems. PhD thesis, Katholihe Universiteit Leuven.

[26] J. Verschelde(1999). Algorithm 795: PHCpack:Ageneral-purpose solver for polynomial systems by homotopy continuation. ACM Trans. on Math. Software, 25(2), 251- 276.

Shawki AL Rashed,

Department of Mathematics,

University of Damascus,

Syria.

Email: rashed@mathematik.uni-kl.de

Gerhard Pfister,

Department of Mathematics,

University of Kaiserslautern,

Germany.

Email: pfister@mathematik.uni-kl.de 
